Article

\title{
The Persuasive Roles of Digital Games: The Case of Cancer Games
}

\author{
Teresa de la Hera Conde-Pumpido \\ Department of Media and Communication, Erasmus University Rotterdam, 503062 PA Rotterdam, Netherlands; E-Mail: \\ delahera@eshcc.eur.nl
}

Submitted: 31 December 2017 | Accepted: 16 March 2018 | Published: 7 June 2018

\begin{abstract}
Using behavioral scientist B. J. Fogg's conceptual framework on the role computer technology plays for users as a starting point, this article argues that persuasion through digital games can be approached from three different perspectives: digital games as media for persuasion, digital games as tools for persuasion and digital games as social actors for persuasion. In this article, I use five cancer gaming cases to illustrate how these three different persuasive roles can be used to accomplish different persuasive goals. In this respect, I explain how each of these persuasive roles digital games can play in the process of persuasion can serve to support cancer patients to face three different challenges: (1) lack of information about the treatment or the disease itself, (2) lack of motivation to start or continue with the treatment, and (3) difficulties in coping with the treatment or the disease. The analysis of these games is theoretical in nature and is done to illustrate my arguments. The categorization proposed in this article can be used as an analytical approach for the study of persuasive gaming strategies.
\end{abstract}

\section{Keywords}

cancer games; persuasion; persuasive games; persuasive technology; serious games; theoretical model

Issue

This article is part of the issue "Games Matter? Current Theories and Studies on Digital Games", edited by Julia Kneer (Erasmus University Rotterdam, The Netherlands) and Ruud Jacobs (University of Twente, The Netherlands).

(C) 2018 by the author; licensee Cogitatio (Lisbon, Portugal). This article is licensed under a Creative Commons Attribution 4.0 International License (CC BY).

\section{Introduction}

The persuasive potential of digital games has been proven to be useful to change, reinforce or shape the attitude and/or behavior of players in several fields such as advertising (Deal, 2005; Wise, Bolls, Kim, Venkataram, \& Meyer, 2008), pro-social communication (Ruggiero, 2013) and healthcare (Orji, Mandryk, Vassileva, \& Gerling, 2013). However, if we pay attention to the different academic definitions used for the concept of persuasive games, and the different categories of persuasive goals studied within this field, it can be concluded that researchers mean different things when they refer to the persuasive potential of digital games.

Persuasive games have been defined as "games that mount procedural rhetoric effectively" (Bogost, 2007b, p. 46), "games explicitly created to change attitudes and behavior" (Ruggiero, 2013, p.213), "digital games that aim to shape, reinforce or change the perceptions, emotions, beliefs, behavioral intentions and behaviors of players" (De la Hera Conde-Pumpido, 2014, p. 75), "games that aim to increase players' awareness of critical and timely social issues" (Kaufman, Flanagan, \& Seidman, 2015, p.174), or "designed interventions with the primary purpose of changing a user's behavior or attitude in an intended way" (Orji et al., 2013). It follows that, while some researchers relate persuasion through digital games to their capacity to convey persuasive messages (Bogost, 2007a), others focus on the games' capacity to trigger specific behaviors (Orji et al., 2013) or facilitating specific interactions among players (Kulyk, Den Daas, David, \& Van Gemert-Pijnen, 2015).

These differences in the way persuasion through digital games is defined and studied are the result of the complex nature of this practice. This complexity is not only due to the wide range of possible applications but the complexity of the process of persuasion itself, and how the specificities of digital games have an influence on this process. For this reason, it is necessary to provide theoretical frameworks that can serve not only to ana- 
lyze the use of different persuasive strategies in relation to different persuasive goals, but that also can be useful tools to select a specific persuasive approach when designing persuasive games. The provision of new theoretical concepts and frameworks is of special relevance in the relatively new field of persuasive games, as these become relevant tools for scholars in the field that can serve to better structure their work and communicate about their insights. This is, therefore, the main goal of this article.

Here, I use an overarching definition of the term persuasive games that serves as an umbrella to all the definitions previously discussed, and that allows me to study this practice from a broad perspective. A persuasive game is understood in this article as a "digital game in which the primary intention of its development is to shape, change or reinforce attitudes toward a concept or object that is separate from the game to match an attitude goal state" (Jacobs, 2017, p. 15). Jacobs suggests this definition in the line of current persuasion research that assumes that behavioral change is always a result or consequence of attitudinal change (O'Keefe, 2002).

To propose the theoretical framework presented in this article, I use as a starting point the conceptual framework of behavioral scientist B. J. Fogg (2003), who described the overlap between persuasion and interactive technology, to argue that it is possible to identify three different roles of digital games when used with persuasive intentions. According to Fogg's conceptual framework, digital games can be used: (1) as media for persuasion providing compelling meaningful experiences that convey specific messages, (2) as tools for persuasion to persuade players in a number of ways such as making a target behavior easier to perform, and ( 3 ) as social actors for persuasion "by applying the same persuasion principles that humans use to influence others" (2003, p. 89). In sections 3-5, I explain in detail how digital games can play these three different roles to influence the attitude of players in different ways. I also explain how the specificities of digital games can be linked to specific persuasive roles, which is something Fogg's framework-based on the role computer technology plays for users-does not address.

In order to illustrate my arguments, I will analyze how the three roles of digital games have been used in the field of healthcare, and specifically for the design of cancer games. In this article, I will use five cancer games as examples of how this topic can be addressed from different approaches when different persuasive roles of digital games are used to support cancer patients.

\section{Digital Games to Support Cancer Patients}

The positive effects of the use of digital games in the field of healthcare is a reality demonstrated by numerous research studies worldwide (Kato, 2010). Digital games are being used with excellent results to, among other things, overcome phobias (Miloff et al., 2016), support rehabili- tation therapies (Reid, 2002) and carry out neuropsychology therapies (Hardy, Bonner, \& Willard, 2010). Among all their applications in the field of healthcare, their use to support cancer patients (especially children and adolescents) stands out.

According to the World Health Organization, cancer is one of the leading causes of mortality worldwide, with 14 million new patients in 2012-a figure that is expected to increase by about $70 \%$ over the next two decades. There is extensive knowledge about the causes of cancer, how it can be prevented, and how to manage the disease once it has been detected. Healthcare persuasion uses this knowledge to promote specific sick-role behaviors. Sick-role behaviors are those undertaken by cancer patients to succeed in their recovery or to improve their quality of life while under treatment (Baranowski et al., 2015). Healthcare persuasion to promote sick-role behaviors in cancer patients includes strategies to help patients adhere to their treatments (e.g. taking a full course of oral chemotherapy), and to manage their disease, e.g., controlling anxiety, reducing stress, eating a balanced diet (Orji et al., 2013).

Previous research has shown that digital games are an effective vehicle for cancer-related healthcare persuasive strategies (Kato, 2010). Cancer treatments, especially chemo, are really aggressive, with many side effects that are not always easy to cope with. For this reason, and especially in the case of children, supporting treatment adherence becomes especially relevant. In this respect, there are three different challenges that patients face: (1) lack of information about the treatment or the disease itself, (2) lack of motivation to start or continue with the treatment, and (3) difficulties in coping with the treatment or the disease. In the following sections, I explain in more detail what these three different challenges mean for cancer patients, and I argue how digital games can play different roles in supporting cancer patients to face each of them. The analysis of the games discussed in the following sections is theoretical in nature and it is done to illustrate my arguments on the different roles digital games can play in the process of persuasion.

\section{Digital Games as Media for Persuasion}

The first challenge of cancer patients that I want to discuss in this article is the lack of information about the treatment, i.e., how it works and what effects it may have, or the disease itself. There are numerous studies that show that many cancer patients have a misperception about the disease or the effects of the treatment before starting with it (Wakefield, Butow, Fleming, Daniel, \& Cohn, 2012), often fearing worse side effects than they actually experience. For this reason, providing complete and accurate information about the reality is essential for patients to face the disease and to adhere to their treatment (Mitchell, Clarke, \& Sloper, 2006). Overcoming this challenge is more complicated when we talk about children or adolescents than in the case of 
adults (Mitchell et al., 2006). A conversation with a doctor or caregiver to talk about the disease, the treatment and its consequences is usually not easy and understandable enough. That is why looking for creative ways to offer treatment information that is understandable to children/adolescents and does not make them afraid is of particular relevance to healthcare specialists. Digital games have proved to be an interesting media to transmit the desired information to children in a way that is well received by them (Kurt \& Savaser, 2011).

In their role as media for persuasion, digital games are used to convey a persuasive message focused on changing the attitude of players by means of persuasive communication. According to the communication scholar Gerald R. Miller, any message that is aimed to shape, reinforce or change the attitudes or behaviors of receivers is to be considered as persuasive communication (2012, p. 7). Therefore, when used as media for persuasion, digital games convey persuasive messages that intentionally aim to influence attitudes. When used as media for persuasion, digital games can promote treatment adherence by conveying messages focused on helping patients to better understand what are they going through and how the therapies and lifestyles they are being asked to stick to can help them to overcome the disease. As previously stated, complete and accurate information supports patients to have a more positive attitude toward treatment adherence (Mitchell et al., 2006). According to Fogg's (2003) theoretical framework, digital games can be used as media for persuasion by: (1) allowing people to explore cause-effect relationships, (2) providing people with vicarious experiences that motivate, or (3) helping people rehearse a behavior.
An example of how digital games have already been used for this purpose is the Re-Mission Game (Hope-Lab, 2006)-see Figure 1-designed to increase knowledge about chemotherapy, change patients' perceptions of their ability to influence health outcomes, and to provide patients with confidence in their ability to meet the specific demands of cancer treatment and recovery. In the game, children control a character that navigates through the interior of the human body. Through the character, they can destroy cancer cells and defeat the main tumor. Thanks to a friendly design and simple game mechanics, children can understand a complex process.

This game acts as a medium for persuasion by allowing children to explore the cause-effect relationships of chemotherapy treatments. The mechanics of the game, and the challenges they need to complete, allow children to explore the effects of chemotherapy on their bodies through their actions in the game. By better understanding how this process works, they can be more open to undergoing their treatment, regardless of the side effects the treatment has on their bodies.

Besides this, being in control of the main character, which allows the children to navigate through their bodies and kill cancer cells to defeat the disease, puts them in control of the situation, and helps them experience the satisfaction of having done something to overcome the disease. This experience can be later translated into their reality: if they undergo the treatment, they are contributing to beating cancer and accordingly playing an important role in this process. The narrative of the game (the role of the main character, which is his/her mission, and the human body as the game space) is therefore also relevant, as it involves the players in a vicarious experi-

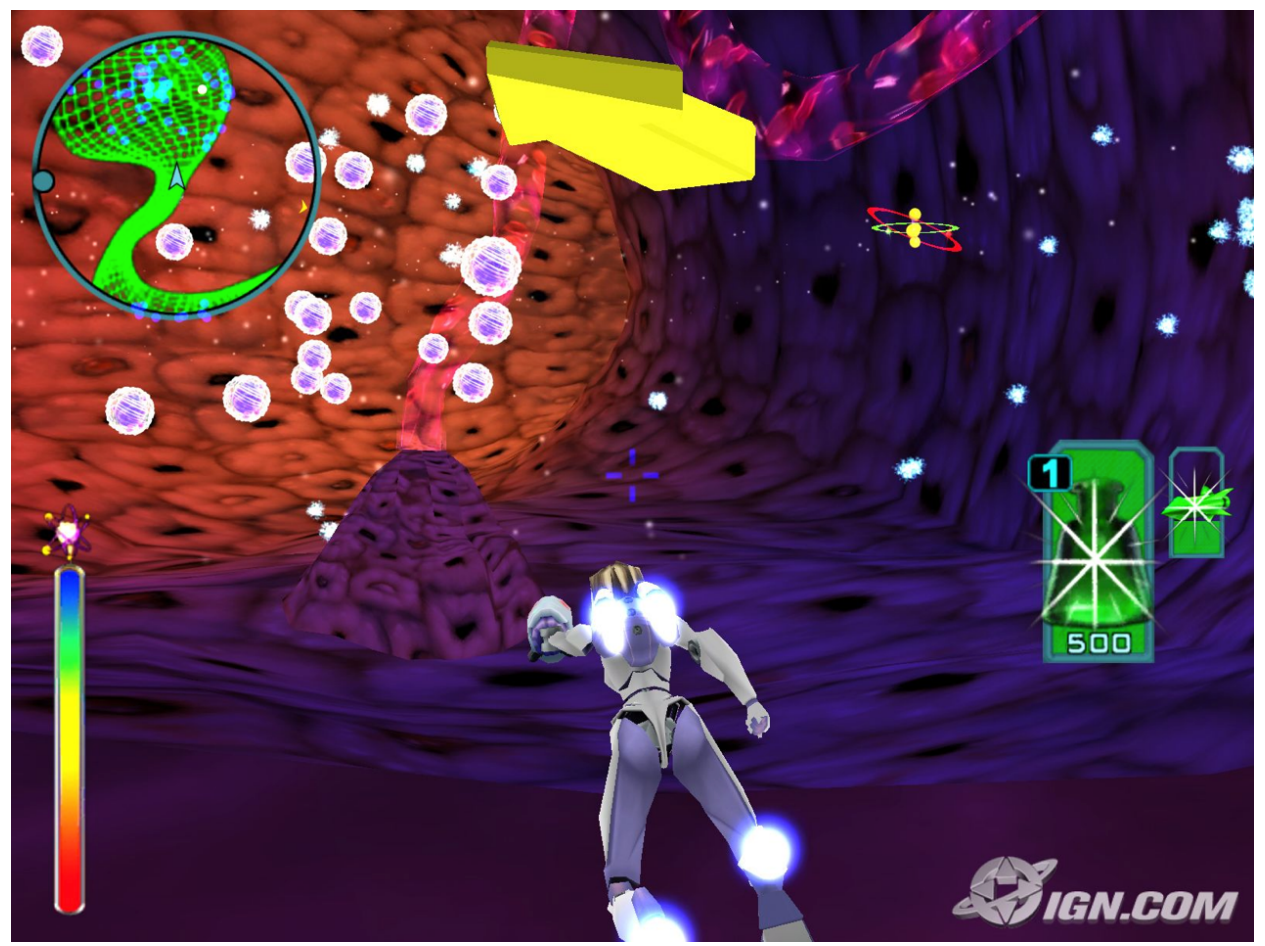

Figure 1. Screenshot of Re-Mission. 
ence that motivates. Research on Re-Mission has indeed shown that it is effective in increasing adherence to treatment among its players (Kurt \& Savaser, 2011).

A second example of how digital games can be used as media for persuasion to overcome cancer patients' lack of information is the Veevia Cancer Game (Wei, 2007), an online game designed to help these patients understand the relevance of following a healthy lifestyle by establishing a relation between healthy living and the efficiency of cancer treatment. The game, designed by the multimedia artist Wei Wei, helps players to comprehend how pathogenic factors can affect organ functions and interfere with treatment efficiency. The player can learn and understand more about the relevance of following a healthy lifestyle by exploring the inner relations between healthy habits and the efficiency of the cancer treatment within the game.

In this game, again, game mechanics are used to help players to explore cause-effect relationships, and the narrative involves them in a vicarious experience designed to motivate them. In Veevia, the player has to explore the human body, with the possibility of different organs that are subject to cancer. Each organ is a new level in the game in which the player needs to face a concrete challenge and can learn about the cause-effect relationship between a specific nocuous habit and the way it interferes with the treatment. In the second level, for example, the player enters the liver (Figure 2). The objective of this level is to make the player understand that drinking alcohol reduces the efficacy of cancer treatment. When the player enters the level, he/she finds a big cancer tumor that blocks his/her way to the door taking him/her to the next level. Quickly, the player understands that he/she needs to kill the tumor to be able to go to the next level. However, if the player tries to use a bunch of medicines (representing chemotherapy treat- ment) placed next to it, they do not seem to have an effect on the tumor. If the player pays attention, he/she discovers that it is possible to choose the drinks that are entering the liver. When the alcoholic drinks are removed, the medicines can be used to kill the tumor. Then the player can easily reach the door that takes him/her to the next level. By completing this level, the player can better understand the relationship between a bad health habit (drinking alcohol) and cancer therapy results. Other levels in the game follow a similar logic. By facing these challenges the player can better comprehend the relationship between a healthy lifestyle and the efficiency of cancer treatments. By rehearsing healthy habits and avoiding nocuous ones in the various levels, the player has time to reflect on the relevance of specific choices and the impact they can have on his/her health.

The two examples discussed in this section illustrate how digital games can be used as media for persuasion to help cancer patients overcome the challenge of lack of information about the disease or the treatment. The brief analysis of these two examples also shows that game mechanics and the narrative are relevant game elements that can be used for the purpose of convening persuasive messages.

\section{Digital Games as Tools for Persuasion}

A second challenge that cancer patients have to face that I want to discuss in this article is related to the lack of motivation to start or continue a treatment. As I mentioned earlier, cancer treatments or their side effects are often aggressive or difficult to cope with. The diagnosis and treatment of cancer is a stressful and threatening experience for patients and especially for children (Hicks \& Lavender, 2001). Although survival rates for cancer are higher than ever before, the course of treatment for can-

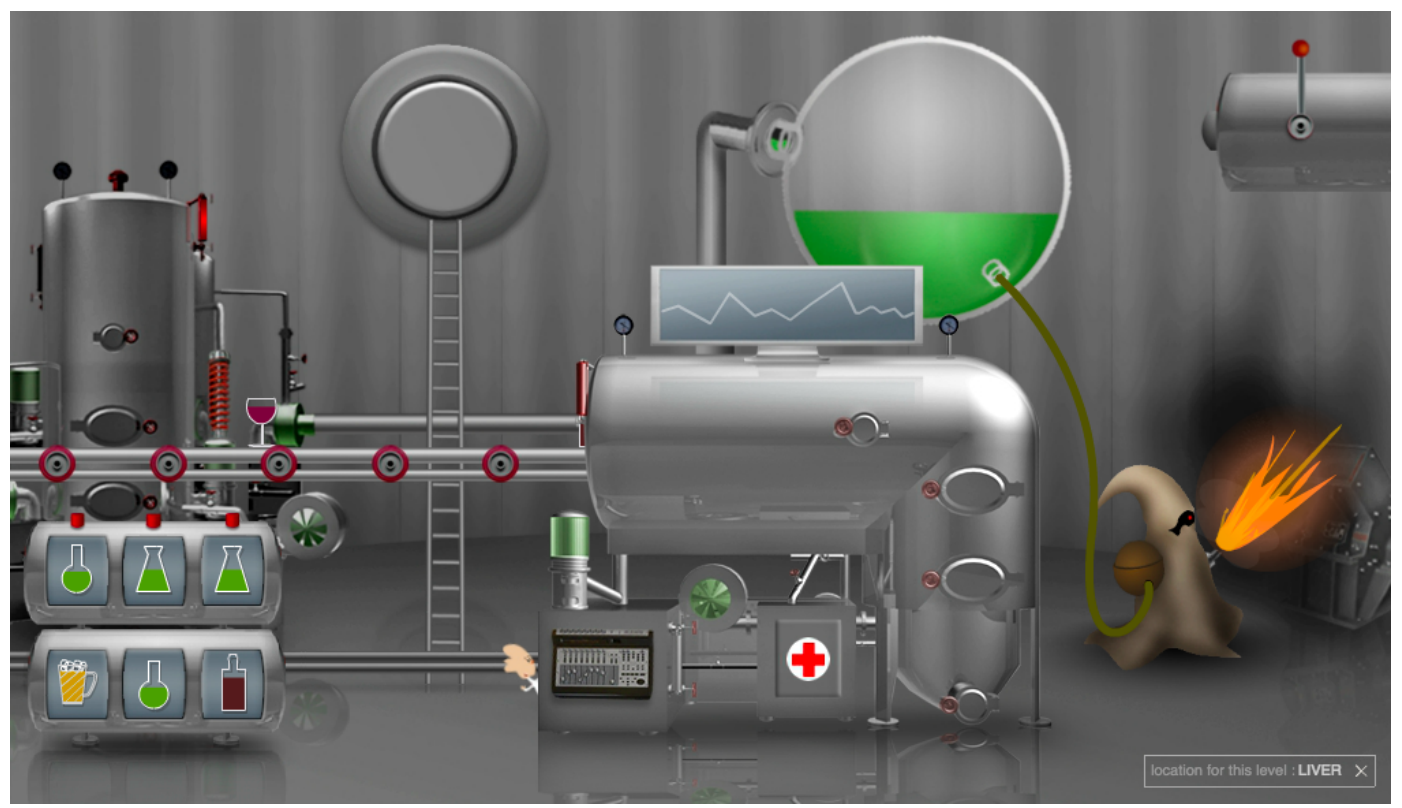

Figure 2. Screenshot of Veevia Cancer Game. 
cer, such as chemotherapy, surgery or radiotherapy, is still a very stressful experience. Cancer patients may not only be at risk from adverse medical effects, but their psychosocial well-being may also be severely affected as a result of cancer and its therapy (Langeveld, Grootenhuis, Voûte, \& de Haan, 2004). For this reason, even if patients have enough information on how a treatment works and its benefits, they may not have sufficient motivation to start or continue with it at a given time. In this section, I want to argue that digital games can help in this regard, serving as a tool to motivate patients to comply with treatment guidelines.

During the past decade, there has been an increase in the use of therapeutic play intervention to help cancer patients cope with the stress of hospitalization and treatment (Li, Chung, \& Ho, 2011). Therapeutic play is a set of structured activities designed according to the psychosocial and cognitive development of cancer patients, as well as health-related issues, to help them cope with psychological and emotional difficulties (LeVieux-Anglin \& Sawyer, 1993). The central goal of therapeutic play is to facilitate the emotional and physical well-being of patients (Vessey \& Mahon, 1990). In their role as tools for persuasion, digital games are designed to influence and motivate people in specific ways by making activities easier or more efficient to do (Fogg, 2003). The games are therefore not used as a medium to convey a persuasive message, but as mediating tools that transform the way patients have to do certain activities in something more attractive to them. Patients are often required to undergo procedures or engage in behaviors that are either painful and aversive (e.g., undergoing chemotherapy), or boring and mundane (e.g., taking pills, exercising on a regular basis). These procedures and behaviors are often necessary to maintain and improve health or even to cure the patient's disease (Kato, 2010, p. 113). When used as tools for persuasion, digital games persuade players to change their attitude toward their treatment by transforming the treatment into something different and more appealing to them.

According to Fogg's theoretical framework (2003), in their role as tools for persuasion, digital games can be used to support cancer patients in undergoing painful or boring procedures by: (1) reducing a complex activity to a few simple steps, (2) leading users through a predetermined sequence of actions or events, step by step, (3) suggesting a behavior at the most opportune moment, (4) helping people to monitor themselves to modify their attitudes or behaviors to achieve a predetermined goal or outcome, or (5) allowing doctors to monitor the behavior of patients.

An example of this is the game Cogmed Memory Training (Pearson, n.d.)-see Figure 3-developed to aid the treatment adherence of patients who experience cognitive problems, which is one of the side effects of treatments such as brain chemotherapy. Neuropsychological rehabilitation treatments require the patient to repeat a series of simple tasks, such as associating objects of the same color and shape, in a continuous and intense way. The treatment can be really exhausting for patients, and it can become boring for children. For this reason, neuropsychologists are using games that help to work the same skills in a more entertaining way for patients. The game Cogmed Memory Training has been shown to be an effective tool in this regard, to support treatment adherence (Cox et al., 2015).

Going back to Fogg's theoretical framework, the game helps to lead patients through the different steps of the treatment through a series of mini-games, focused on training different skills in patients that are necessary for their recovery. The game keeps patients busy and focused on different challenges and levels, which help them to persist in the treatment. Besides this, the game helps patients and doctors to monitor their progress, which helps patients increase their perception of being in control of their own health, and also helps doctors

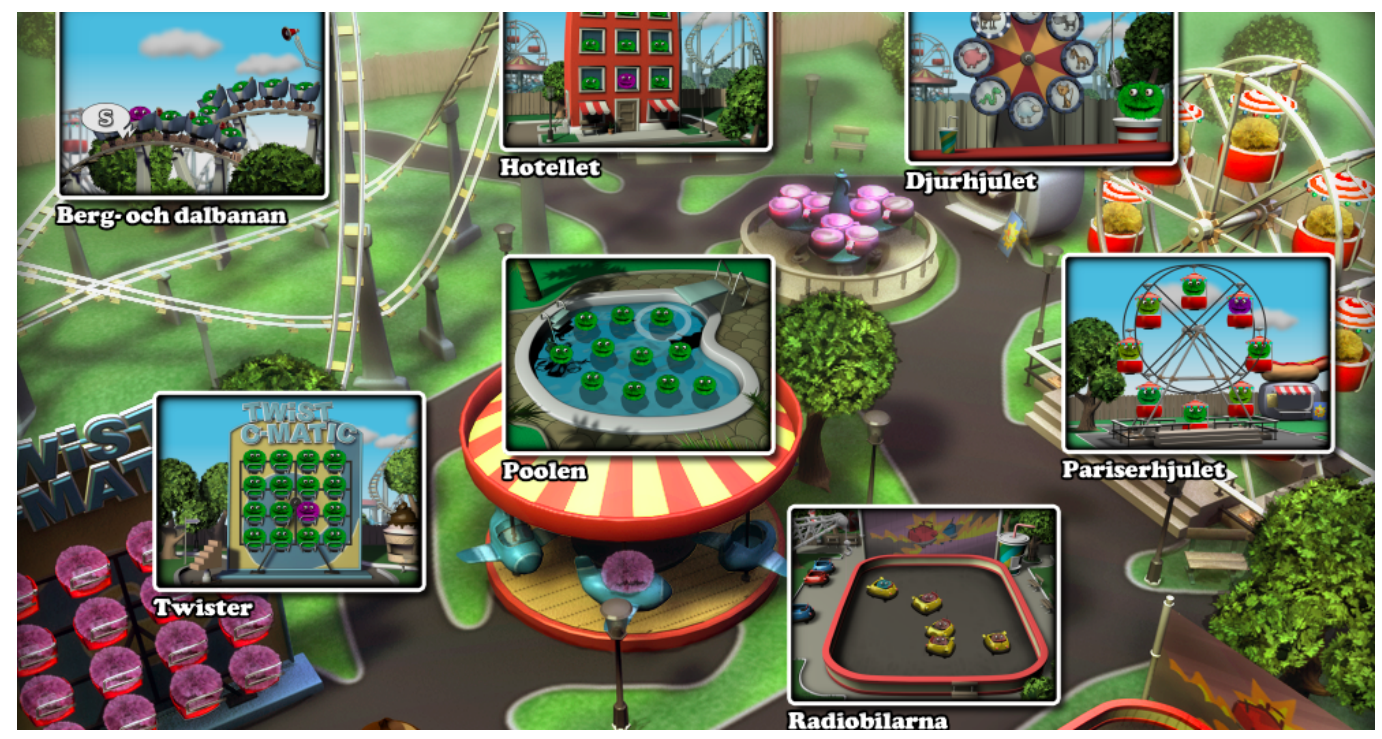

Figure 3. Screenshot of Cogmed Memory Training. 
to adapt the treatment considering patients' progress. What makes this game an efficient tool to influence players' attitude toward the treatment is what I call tactical persuasion (De la Hera Conde-Pumpido, 2014), which is using the rules of the game and the game mechanics to engage players in the game session, and to motivate them to start playing the game and to keep playing it. Besides this, the feedback provided to the player and the doctor on the progress of the patient is also of special relevance and key to motivating the player to continue with the treatment.

The example discussed in this section show how digital games, in their role as tools for persuasion, can motivate cancer patients to start or continue with a treatment that might be difficult to cope with. In this case, the rules of the game are crucial to motivate and engage players in specific behaviors, and the feedback provided throughout the game also becomes of special relevance.

\section{Digital Games as Social Actors for Persuasion}

A third challenge that cancer patients face that I want to discuss in this article is the problems in coping with the disease or the treatment and its side effects. Some patients have all the information about the treatment, and have the motivation to carry it out, but have difficulties coping. Examples of these difficulties include anxiety or fear of how the disease will develop, discomfort generated by the treatment received, and hair loss in the case of patients treated with chemotherapy. In this respect, the support of other patients who are going through or have experienced the same difficulties can be of particular help. However, it is not always easy to establish new relationships with other patients, or initiate conversations on issues that concern you with people with whom you do not have a close relationship. To support patients to face this challenge, digital games can take the role of social actors for persuasion and be used to mediate the process of seeking social support. The persuasive potential of this role relies on the capacity of digital games to be used to persuade players, "by giving a variety of social cues that elicit social responses" from them (Fogg, 2003, p. 89).

When used as social actors for persuasion, digital games may utilize either computer-human persuasion or computer-mediated persuasion (Oinas-Kukkonen \& Harjumaa, 2008). On the one hand, when used as a form of computer-human persuasion, digital games may utilize some patterns of interaction similar to social communication (Fogg \& Nass, 1997). This is the form of persuasion through computers that is addressed by Fogg (2003) when he discusses the role of technologies as social actors for persuasion. In this article, however, I also want to discuss the possibility of digital games to use computer-mediated persuasion in their role as social actors for persuasion. Computer-mediated persuasion means that people are persuading others through computers, e.g. discussion forums, e-mail, instant messages, blogs, or social network systems (Oinas-Kukkonen \& Har- jumaa, 2008). Recent research, for example, shows that dialogue support features play a significant role in relation to adherence to web-based health interventions, especially for young adults, who tend to be influenced by their peers (Kulyk et al., 2015).

An example of how digital games can use computerhuman persuasion to support cancer patients is Aflac Duck (see Figure 4). Aflac Duck is a robot duck designed to help and entertain children going through chemotherapy to cope with cancer. The duck comes accompanied by a tablet game children can use to interact and play with him. Using the table game and a built-in little chemotherapy port, children can put their duck companion through rounds of chemo, making real treatment a little less scary. As part of the game, children should also feed Aflac, bathe him or dance with him, among other things. This helps reverse the roles of the children from suffering patients to helping caregivers, thus giving them control of their experience, which helps to change their attitude toward treatment.

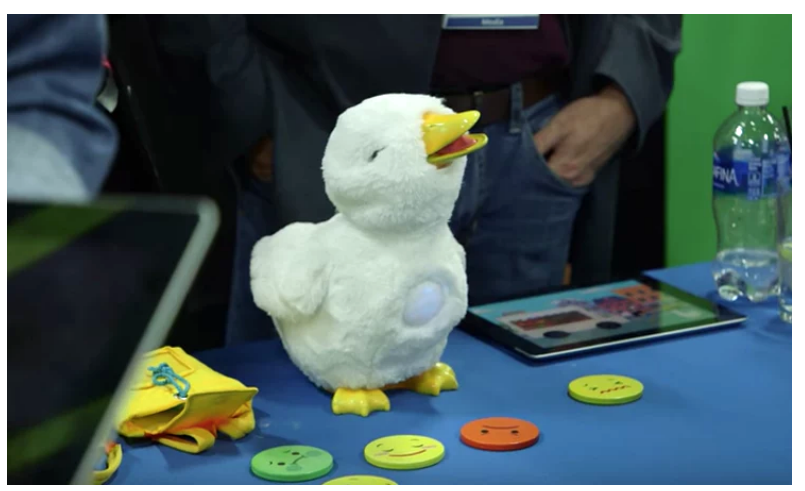

Figure 4. Aflac Duck.

Through the emotional and interactive responses, Aflac is perceived as a social actor that according to Fogg (2003) is able to leverage the principles of social influence that serve to motivate and persuade. Through the embodied character, different types of social cues can result in social influence. Physical cues such as Aflac's body movements and facial expressions are clearly used in the game to communicate with players. The main goal is to help children feel they are not alone in the process. The duck comes with several emoji counters with feelings children experience most while undergoing treatmenthappy, silly, scared, nauseous, angry, calm, and sadwhich kids can tape to the duck's chest and watch it act out, helping them express and share their feelings. Through these cues, Aflac conveys the pain and difficulties he is going through at certain moments (for example, when chemotherapy is administered), but moreover he helps children to reflect on the relevance of hope and persistence. Psychological cues are also relevant in the game; how feelings and emotions are communicated through the character, by the use of physical expression or language, become relevant. 
As stated before, besides using computer-human persuasion, digital games can also use computer-mediated persuasion in their role as social actors for persuasion. This means that the game becomes a mediating tool between different players, facilitating some players to persuade other players. An example of how digital games can play the role of social actors for persuasion by using computer-mediated persuasion is the platform Adventures in Sophoria developed at the University of Duisburg-Essen with the goal of facilitating the intercommunication of teenagers during cancer treatment. The game becomes the point of union between players, who begin to talk about their game and the characters of their game, and on many occasions that first contact evolves until a real friendship is established between two people who are going through similar difficulties, thus delivering a support for coping. This is related to the capacity of digital games to foster social interaction (De la Hera Conde-Pumpido \& Paz Aléncar, 2015), and the tools implemented in the game itself to facilitate this interaction, such as the chat box that allows players to chat while playing or the options to share links and private messages with their friends. Besides this, the context in which these games are played-a Massively Multiplayer Online Role Playing Game (MMORPG) especially aimed at young cancer patients-becomes relevant in this process, as users might find it a natural context to look for contact with other people going through similar experiences, which can help them to feel more comfortable about disclosing their personal experiences, sharing emotions and feelings, and looking for support. We can then talk about game-mediated type of persuasion, meaning that the game becomes persuasive when being played in a specific context with a specific purpose (De la Hera Conde-Pumpido, 2017).

The two examples discussed in this section serve to illustrate two different ways in which digital games can play the role of social actors for persuasion to support cancer patients. This shows the differences between computer-human persuasion and computer-mediated persuasion. In the first case, we can see how embodied characters in games, how they are represented, how they interact with other characters with a specific context, and how players can influence their performance in the game through interactions, play important roles in the process of human-computer interaction. In the case of computer-mediated interaction, social persuasion, which is the capacity of digital games to foster social interactions (De la Hera Conde-Pumpido, 2014), becomes more relevant.

\section{Conclusions}

The five games analyzed in this article are just some examples of how digital games can help overcome the three challenges related to adherence to cancer treatments. Through these cases, I have illustrated how digital games can play three different roles in the process of persuasion, becoming media, tools and social actors for persuasion. The discussion of these examples serves to illustrate how the theoretical framework presented in this article can help to better understand how the different roles that digital games can play in the process of persuasion can achieve different goals when games are used for persuasive purposes. This is useful to analyze how different persuasive strategies are being used to achieve different persuasive goals and how different game elements better respond to different persuasive intents. Besides, this framework can be an interesting tool for designers of persuasive games, so they can make informed decisions when choosing a persuasive strategy for their designs. I am currently working on a theoretical model that links the use of the different persuasive roles to specific aims of the game. This model will serve to further explain how these different roles can better respond to different persuasive challenges such as changing, shaping or reinforcing the attitude of players. Additionally, this model will link these two variables to a third one related to the level of involvement of players in the process of persuasion.

\section{Acknowledgements}

This work is part of the research programme "Persuasive Gaming in Context: From Theory-Based Design to Validation and Back" with project number 314-99-106 which is financed by the Netherlands Organisation for Scientific Research (NWO). A previous version of this article was published in GALA Conference 2017 Proceedings. The article was revised and extended for this publication.

\section{Conflict of Interests}

The author declares no conflict of interests.

\section{References}

Baranowski, T., Blumberg, F., Buday, R., DeSmet, A., Fiellin, L. E., Green, C. S., . . . Young, K. (2015). Games for health for children-Current status and needed research. Games for Health Journal, 5(1), 1-12. doi:10.1089/g4h.2015.0026

Bogost, I. (2007a). Persuasive games: The expressive power of videogames. Cambridge, MA: MIT Press.

Bogost, I. (2007b). Persuasive games on mobile devices. In B. J. Fogg \& D. Eckles (Eds.), Mobile persuasion. Stanford: Stanford University. Retrieved from http://www.bogost.com/downloads/I.\%20Bogost\% 20Mobile\%20Persuasion.pdf

Cox, L. E., Ashford, J. M., Clark, K. N., Martin-Elbahesh, K., Hardy, K. K., Merchant, T. E., . . . Conklin, H. M. (2015). Feasibility and acceptability of a remotely administered computerized intervention to address cognitive late effects among childhood cancer survivors. Neuro-Oncology Practice, 2(2), 78-87. doi:10.1093/nop/npu036 
Deal, D. (2005). The ability of branded online games to build brand equity: An exploratory study. In DiGRA 2005 conference: changing views-worlds in play. Vancouver: DiGRA.

De la Hera Conde-Pumpido, T. (2014). Persuasive structures in advergames: Conveying advertising messages through digital games. Utrecht: Utrecht University.

De la Hera Conde-Pumpido, T. (2017). Persuasive gaming: Identifying the different types of persuasion through games. International Journal of Serious Games, 4(1), 31-39. doi:10.17083/ijsg.v4i1.140

De la Hera Conde-Pumpido, T., \& Paz Aléncar, A. (2015). Collaborative digital games as mediation tool to foster intercultural integration in primary Dutch schools. E-Learning Papers, 43, 13-23.

Fogg, B. J. (2003). Persuasive technology using computers to change what we think and do. San Francisco, CA: Elsevier.

Fogg, B. J., \& Nass, C. (1997). How users reciprocate to computers: An experiment that demonstrates behavior change. Chi 97, (March), 331-332. doi.org/ 10.1145/1120212.1120419

Hardy, K., Bonner, M., \& Willard, V. (2010). Computerized cognitive training for survivors of pediatric cancer. $P e$ diatric Blood and Cancer, 55(5), 776-777.

Hicks, M. D., \& Lavender, R. (2001). Psychosocial practice trends in pediatric oncology. Journal of Pediatric Oncology Nursing, 18(4), 143-153. doi:10.1053/ jpon.2001.24795

Jacobs, R. S. (2017, November 10). Playing to win over: Validating persuasive games (Dissertation). ERMeCCErasmus Research Center for Media Communication and Culture, Rotterdam, Netherlands. Retrieved from https://repub.eur.nl/pub/102769

Kato, P. M. (2010). Video games in health care: Closing the gap. Review of General Psychology, 14(2), 113-121. doi:10.1037/a0019441

Kaufman, G., Flanagan, M., \& Seidman, M. (2015). Creating stealth game interventions for attitude and behavior change: An "Embedded Design" model. In Proceedings of DiGRA 2015: Diversity of play: games-cultures-identities (pp. 1-13). Lüneburg, Germany: University of Lüneburg.

Kulyk, O., Den Daas, C., David, S., \& Van Gemert-Pijnen, L. (2015). How persuasive are serious games, social media and mHealth technologies for vulnerable young adults? Design factors for health behavior and lifestyle change support: Sexual health case. In CEUR Workshop Proceedings, 1369, 28-42.

Kurt, A. S., \& Savaser, S. (2011). An effect of re-mission video game on perceived stress levels of adolescents with cancer. Acta Paediatrica, International Journal of Paediatrics, 100, 98-99. doi:10.1111/j.16512227.2011.02488.x

Langeveld, N. E., Grootenhuis, M. A., Voûte, P. A., \& de Haan, R. J. (2004). Posttraumatic stress symptoms in adult survivors of childhood cancer. Pediatric Blood \& Cancer, 42, 604-610. doi:10.1002/pbc.20024
LeVieux-Anglin, L., \& Sawyer, E. H. (1993). Incorporating play interventions into nursing care. Pediatric Nursing, 19(5), 459-463.

Li, W. H. C., Chung, J. O. K., \& Ho, E. K. Y. (2011). The effectiveness of therapeutic play, using virtual reality computer games, in promoting the psychological wellbeing of children hospitalised with cancer. Journal of Clinical Nursing, 20(15/16), 2135-2143. doi:10.1111/ j.1365-2702.2011.03733.x

Miller, G. R. (2012). On being persuaded: Some basic distinctions. In J. P. Dillard \& L. Shen (Eds.), The SAGE handbook of persuasion: Developments in theory and practice (pp. 70-82). doi:10.4135/ 9781452218410.n5

Miloff, A., Lindner, P., Hamilton, W., Reuterskiöld, L., Andersson, G., \& Carlbring, P. (2016). Single-session gamified virtual reality exposure therapy for spider phobia vs. traditional exposure therapy: Study protocol for a randomized controlled non-inferiority trial. Trials, 17(1), 1-8. doi:10.1186/s13063-016-1171-1

Mitchell, W., Clarke, S., \& Sloper, P. (2006). Care and support needs of children and young people with cancer and their parents. Psycho-Oncology, 15(9), 805-816. doi:10.1002/pon.1014

Oinas-Kukkonen, H., \& Harjumaa, M. (2008). Towards deeper understanding of persuasion in software and information systems. In Proceedings of the 1st international conference on advances in computer-human interaction, ACHI 2008 (pp. 200-205). doi:10.1109/ ACHI.2008.31

O'Keefe, D. J. (2002). Persuasion: Theory and research. Current Directions in Psychological Science, 16, 1-384.

Orji, R., Mandryk, R. L., Vassileva, J., \& Gerling, K. M. (2013). Tailoring persuasive health games to gamer type. Proceedings of the SIGCHI conference on human factors in computing systems-CHI '13, 2467-2476. doi:10.1145/2470654.2481341

Reid, D. T. (2002). Benefits of a virtual play rehabilitation environment for children with cerebral palsy on perceptions of self-efficacy: a pilot study. Pediatric Rehabilitation, 5(3), 141-148. doi:10.1080/ 1363849021000039344

Ruggiero, D. (2013). The effect of a persuasive game on attitude towards the homeless (Unpublished thesis). Purdue University, West Lafayette, USA.

Vessey, J. A., \& Mahon, M. M. (1990). Therapeutic play and the hospitalized child. Journal of Pediatric Nursing, 5(5), 328-333.

Wakefield, C. E., Butow, P., Fleming, C. A. K., Daniel, G., \& Cohn, R. J. (2012). Family information needs at childhood cancer treatment completion. Pediatric Blood and Cancer, 58(4), 621-626. doi:10.1002/pbc.23316

Wise, K., Bolls, P. D., Kim, H., Venkataram, A., \& Meyer, R. (2008). Enjoyment of advergames and brand attitudes: The impact of thematic relevance. Journal of Interactive Advertising, 9(1), 27-36. 


\section{About the Author}

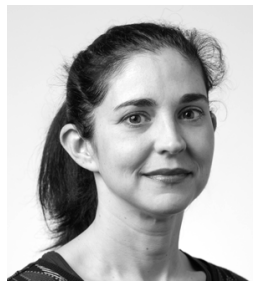

Teresa de la Hera is a Lecturer and Researcher in the Department of Media \& Communication at Erasmus University Rotterdam, in the Netherlands. She has expertise in the fields of serious games and new media. She started her academic career at the University of Santiago de Compostela in Spain in 2006. In 2011, she moved to the Netherlands, where she finished her PhD and was a Post-Doctoral Researcher and Lecturer at the Department of Media and Culture Studies at Utrecht University until 2017. 